\title{
A. HORN'S RESULT ON MATRICES WITH PRESCRIBED SINGULAR VALUES AND EIGENVALUES*
}

\author{
TIN-YAU TAM ${ }^{\dagger}$
}

\begin{abstract}
We give a new proof of a classical result of A. Horn on the existence of a matrix with prescribed singular values and eigenvalues.
\end{abstract}

Key words. Eigenvalues, singular values.

AMS subject classifications. 15A45, 15A18.

Let $A \in \mathbb{C}_{n \times n}$ and let $\lambda_{1}, \ldots, \lambda_{n}$ be the eigenvalues of $A$ arranged in the order $\left|\lambda_{1}\right| \geq \cdots \geq\left|\lambda_{n}\right|$. The singular values of $A$ are the nonnegative square roots of the eigenvalues of the positive semi-definite matrix $A^{*} A$ and are denoted by $s_{1} \geq \cdots \geq s_{n}$. Weyl's inequalities [7] provide a very nice relation between the eigenvalues and singular values of $A$ :

$$
\begin{aligned}
& \prod_{j=1}^{k}\left|\lambda_{j}\right| \leq \prod_{j=1}^{k} s_{j}, \quad k=1, \ldots, n-1, \\
& \prod_{j=1}^{n}\left|\lambda_{j}\right|=\prod_{j=1}^{n} s_{j} .
\end{aligned}
$$

The equality follows from two ways of expressing the absolute value of the determinant of $A$. A. Horn [2] established the converse of Weyl's result.

TheOREm 1.1. (A. Horn) If $\left|\lambda_{1}\right| \geq \cdots \geq\left|\lambda_{n}\right|$ and $s_{1} \geq \cdots \geq s_{n}$ satisfy (1.1) and (1.2), then there exists $A \in \mathbb{C}_{n \times n}$ such that $\lambda_{1}, \ldots, \lambda_{n}$ are the eigenvalues and $s_{1}, \ldots, s_{n}$ are the singular values of $A$.

Horn's original proof is divided into two cases: (i) $s_{n} \neq 0$ (the nonsingular case) and (ii) $s_{n}=0$ (the singular case. There is a typo: $C_{m, m+1}=\gamma$ and $C_{i, i+1}=\alpha_{i}$ should be $C_{m+1, m}=\gamma$ and $C_{i+1, i}=\alpha_{i}$ on $[2$, p.6]). In this note we provide a new proof of Horn's result. Our proof differs from Horn's proof in two ways that (i) our proof is divided into two cases according to $\lambda_{1}=0$ and $\lambda_{1} \neq 0$, and (ii) our induction technique is different. It is very much like Chan and Li's technique [1] (the same

\footnotetext{
${ }^{*}$ Received by the editors on June 10, 2009. Accepted for publication on July 31, 2010. Handling Editors: Roger A. Horn and Fuzhen Zhang.

$\dagger^{\dagger}$ Department of Mathematics and Statistics, Auburn University, AL 36849-5310, USA (tamtiny@auburn.edu).
} 
technique is also used in [8]) for proving another result of Horn [3] (on the diagonal entries and eigenvalues of a Hermitian matrix): ours is multiplicative and Chan and Li's is additive. See [4, Section 3.6] for a proof of Theorem 1.1 using the result of Horn [3]. Also see [5] for an extension of Weyl-Horn's result and a numerically stable construction of $A$. A technique similar to that of Chan and Li can be found in Thompson's earlier work [6] on the diagonal entries and singular values of a square matrix.

Proof. We divide the proof into two cases: nilpotent or not.

Case i: $\lambda_{1}=0$. Then $s_{n}=0$ by (1.2) and we choose

$$
A:=\left(\begin{array}{cccc}
0 & s_{1} & & \\
& 0 & \ddots & \\
& & & s_{n-1} \\
& & & 0
\end{array}\right)
$$

Case ii: $\lambda_{1} \neq 0$. We will use induction on $n$. When $n=2$, the matrix

$$
A=\left(\begin{array}{cc}
\lambda_{1} & \mu \\
0 & \lambda_{2}
\end{array}\right)
$$

has singular values $s_{1} \geq s_{2}$ if we set

$$
\mu:=\left(s_{1}^{2}+s_{2}^{2}-\left|\lambda_{1}\right|^{2}-\left|\lambda_{2}\right|^{2}\right)^{1 / 2} .
$$

Suppose that the statement of Theorem 1.1 is true for $\lambda_{1} \neq 0$ when $n=m \geq 2$. Let $n=m+1$ and let $j \geq 2$ be the largest index such that $s_{j-1} \geq\left|\lambda_{1}\right| \geq s_{j}$. Clearly $s_{1} \geq \max \left\{\left|\lambda_{1}\right|, s_{1} s_{j} /\left|\lambda_{1}\right|\right\} \geq \min \left\{\left|\lambda_{1}\right|, s_{1} s_{j} /\left|\lambda_{1}\right|\right\}$. Then there exist $2 \times 2$ unitary matrices $U_{1}$ and $V_{1}$ such that

$$
U_{1}\left(\begin{array}{cc}
s_{1} & \\
& s_{j}
\end{array}\right) V_{1}=\left(\begin{array}{cc}
\lambda_{1} & \mu^{\prime} \\
0 & s_{1} s_{j} /\left|\lambda_{1}\right|
\end{array}\right),
$$

where $\mu^{\prime}=\left(s_{1}^{2}+s_{j}^{2}-\left|\lambda_{1}\right|^{2}-s_{1}^{2} s_{j}^{2} /\left|\lambda_{1}\right|^{2}\right)^{1 / 2}$. Set $U_{2}:=U_{1} \oplus I_{m-1}, V_{2}:=V_{1} \oplus I_{m-1}$. Then

$$
\begin{aligned}
A_{1} & :=U_{2} \operatorname{diag}\left(s_{1}, s_{j}, s_{2}, \ldots, s_{j-1}, s_{j+1}, \ldots, s_{m+1}\right) V_{2} \\
& =\left(\begin{array}{cc}
\lambda_{1} & \mu^{\prime} \\
0 & s_{1} s_{j} /\left|\lambda_{1}\right|
\end{array}\right) \oplus \operatorname{diag}\left(s_{2}, \ldots, s_{j-1}, s_{j+1}, \ldots, s_{m+1}\right) .
\end{aligned}
$$

It suffices to show that $\left(s_{1} s_{j} /\left|\lambda_{1}\right|, s_{2}, \ldots, s_{j-1}, s_{j+1}, \ldots, s_{m+1}\right)$ and $\left(\lambda_{2}, \ldots, \lambda_{m+1}\right)$ satisfy (1.1) and (1.2). Since $s_{j-1} \geq\left|\lambda_{1}\right| \geq\left|\lambda_{2}\right|$,

$$
\left|\lambda_{2}\right| \leq \max \left\{s_{1} s_{j} /\left|\lambda_{1}\right|, s_{2}, \ldots, s_{j-1}, s_{j+1}, \ldots, s_{m+1}\right\} .
$$


Moreover

$$
\begin{aligned}
& \prod_{i=2}^{k}\left|\lambda_{i}\right| \leq\left|\lambda_{1}\right|^{k-1} \leq \prod_{i=2}^{k} s_{i}, \quad k=2, \ldots, j-1, \\
& \prod_{i=2}^{k}\left|\lambda_{i}\right|=\frac{1}{\left|\lambda_{1}\right|} \prod_{i=1}^{k}\left|\lambda_{i}\right| \leq \frac{s_{1} s_{j}}{\left|\lambda_{1}\right|} \prod_{i=2, i \neq j}^{k} s_{i}, \quad k=j, \ldots, m, \quad \text { by }(1.1) \\
& \prod_{i=2}^{m+1}\left|\lambda_{i}\right|=\frac{s_{1} s_{j}}{\left|\lambda_{1}\right|} \prod_{i=2, i \neq j}^{m+1} s_{i} \quad \text { by }(1.2) .
\end{aligned}
$$

We consider two cases: (a) $\lambda_{2}=0$ and apply Case i. (b) $\lambda_{2} \neq 0$ and apply the induction hypothesis in Case ii. For both cases, there exist $m \times m$ unitary matrices $U_{3}, V_{3}$ such that

$$
U_{3} \operatorname{diag}\left(\frac{s_{1} s_{2}}{\left|\lambda_{1}\right|}, s_{2}, \ldots, s_{j-1}, s_{j+1}, \ldots, s_{m+1}\right) V_{3}
$$

is upper triangular with diagonal $\left(\lambda_{2}, \ldots, \lambda_{m+1}\right)$. Then $A=U_{4} A_{1} V_{4}$ is the desired matrix, where $U_{4}:=1 \oplus U_{3}, V_{4}:=1 \oplus V_{3}$.

Acknowledgment: The author is very thankful to Roger Horn for many valuable comments and suggestions.

\section{REFERENCES}

[1] N.N. Chan and K.H. Li. Diagonal elements and eigenvalues of a real symmetric matrix. J. Math. Anal. Appl., 91 (1983) 562-566.

[2] A. Horn. On the eigenvalues of a matrix with prescribed singular values. Proc. Amer. Math. Soc., 5 (1954) 4-7.

[3] A. Horn. Doubly stochastic matrices and the diagonal of a rotation matrix. Amer. J. Math., 76 (1954), 620-630.

[4] R.A. Horn and C.R. Johnson. Topics in Matrix Analysis. Cambridge Univ. Press, 1991.

[5] C.K. Li and R. Mathias. Construction of matrices with prescribed singular values and eigenvalues. BIT 41 (2001) 115-126.

[6] R.C. Thompson. Singular values, diagonal elements, and convexity. SIAM J. Appl. Math., 32 (1977) 39-63.

[7] H. Weyl. Inequalities between the two kinds of eigenvalues of a linear transformation. Proc. Nat. Acad. Sci. U.S.A., 35 (1949) 408-411.

[8] H. Zha and Z. Zhang. A note on constructing a symmetric matrix with specified diagonal entries and eigenvalues. BIT, 35 (1995) 448-452. 